\title{
Bounding Sequential Estimation Errors Due to Gauss-Markov Noise with Uncertain Parameters
}

\author{
Steven Langel, The MITRE Corporation \\ Omar García Crespillo, Institute of Communications and Navigation, German Aerospace Center (DLR) \\ Mathieu Joerger, The University of Arizona
}

\begin{abstract}
This paper describes the derivation and implementation of a new method to overbound Kalman filter (KF) based estimate error distributions in the presence of time-correlated measurement and process noise. The method is specific to problems where each input noise component is first-order Gauss-Markov with a distinct variance $\sigma^{2} \in\left[\sigma_{\min }^{2}, \sigma_{\max }^{2}\right]$ and time constant $\tau \in\left[\tau_{\min }, \tau_{\max }\right]$. The bounds on $\sigma^{2}$ and $\tau$ are known. Reference [1] derives an overbound for the continuous-time KF, and we extend the result to the more common case of sampled-data systems with discrete-time measurements. We prove that the KF covariance matrix overbounds the estimate error distribution when Gauss-Markov processes are defined using a time constant $\tau_{\max }$ and a process noise variance inflated by $\left(\tau_{\max } / \tau_{\min }\right)$. We also show that the overbound is tightest by initializing the variance of the Gauss-Markov process with $\sigma_{0}^{2}=2 \sigma_{\max }^{2} /\left[1+\left(\tau_{\min } / \tau_{\max }\right)\right]$. The new method is evaluated using covariance analysis for an example application in advanced receiver autonomous integrity monitoring (ARAIM) [2].

The author's affiliation with The MITRE Corporation is provided for identification purposes only and is not intended to convey or imply MITRE's concurrence with, or support for, the positions, opinions, or viewpoints expressed by the author.
\end{abstract}

\section{Introduction}

Safety-critical GNSS applications must ensure that the probability of the estimate error exceeding predefined bounds is acceptably small. This probability is often referred to as integrity risk. In the aviation community, CDF overbounding has been used extensively over the past 10-20 years to derive upper bounds on integrity risk for snapshot least-squares, GPS-based navigation. However, state estimation using a recursive filter can be more accurate and enables integration with additional sensors like an inertial measurement unit (IMU). A specific example where recursive filtering is being explored is Vertical ARAIM, or V-ARAIM, which seeks to provide worldwide vertical guidance of aircraft using dual-frequency measurements from multiple GNSS constellations. It was shown recently that V-ARAIM performance can be improved significantly by processing time-sequences of measurements [3, 4]. A major challenge with sequential estimation is how to conservatively account for time-correlated noise like GPS multipath. Precise multipath models are very difficult to obtain, as illustrated in [5] for an ensemble of empirically derived multipath autocorrelation functions (ACFs). Thus, even using the best models available, there is an element of uncertainty when dealing with time-correlated noise.

Existing CDF overbounding methods do not account for model uncertainty over time, which has prompted the development of new techniques. Theoretical approaches were developed in $[6,7]$ to bound the integrity risk of linear systems driven by correlated noise that is known to be the output of a linear system driven by a spherically symmetric random process. The authors in [8] derive an 
integrity risk bound for the KF when measurement and process noise are Gaussian with ACFs that are unknown but can be upper and lower bounded. The methods in [6-8] are attractive because they do not require any specific knowledge about the mathematical form of the input noise autocorrelation functions. However, the price to pay is that [6-8] all provide a batch solution in the sense that they require storing matrices whose dimensions grow without bound at a rate dictated by sensor sampling rates. For real-time applications, these techniques are only suitable for short duration applications. If high rate sensors like an IMU are being used, the maximum allowable duration could be on the order of minutes before computational and memory constraints begin to prohibit real-time operation. One way to derive a practical algorithm is to stipulate that the measurement and process noise autocorrelation functions are known up to a finite set of uncertain parameters.

State estimation with uncertain parameters has been studied extensively in the robust estimation literature. Guaranteed cost filtering emerged in the 1990s as a technique for defining a robust filter whose estimate error variance is guaranteed to be smaller than a given bound. These filters often require determination of one or more scale parameters using an optimization algorithm and can display unstable behavior. Dependence on scale-parameters and questionable stability characteristics means that significant up-front effort is required to design the estimator. Real-time capability is also a significant challenge given the need to numerically solve an optimization problem. For these reasons, robust estimators have not been adopted by the navigation community to upper bound KF integrity risk when the input noise autocorrelation functions are uncertain. The difficulties just described stem from the requirement that the robust estimator minimize a specified cost function (e.g., minimum mean square error). Practical guaranteed cost estimators can be obtained if the estimator is not required to be optimal.

In [1], a guaranteed upper bound on the KF estimate error variance is derived when process and measurement noise components are first-order Gauss-Markov processes with unknown, but bounded time constants. The authors showed that in order to guarantee that the KF covariance matrix will bound the true position estimate error, both the minimum and maximum time constants must be used in the KF matrices. The derivation is conducted for the continuous time KF and demonstrated for a simplified GPS/IMU navigation problem. Even though [1] does not address the more general problem where the mathematical structure of the input noise ACFs is unknown, it serves as a good starting point that will hopefully stimulate further research on this topic.

The first contribution of this work is to extend the results in [1] to the more common case of sampled-data systems with discrete-time measurements. Second, a performance analysis is carried out for aircraft approach applications that use time-sequential V-ARAIM with GPS and Galileo. Multipath error is first-order Gauss-Markov with an unknown variance and time constant that each reside in a specified interval. The performance metric is availability, defined as the percentage of time over the GPS/Galileo repeatability period where the integrity risk bound meets a predefined requirement. Global availability maps are established for the traditional approach where the upper bound variance and time constant are used in the KF, and for the new approach derived in the paper. We show that when upper bound parameter values are used, availability predictions are optimistic compared to their true value. On the other hand, the new method is shown to produce an availability prediction that is guaranteed to be conservative, even though the multipath variance and time constant are uncertain. 


\section{Problem Setup}

This paper is concerned with state estimation for the hybrid linear system

$$
\begin{aligned}
\dot{\boldsymbol{\xi}} & =\mathbf{A}(t) \xi+\mathbf{B}(t) \boldsymbol{w}(t) \\
\boldsymbol{z}_{k} & =\mathbf{C}_{k} \xi_{k}+\mathbf{D}_{k} \boldsymbol{v}_{k}
\end{aligned}
$$

where $\boldsymbol{\xi} \in \mathbb{R}^{n}$ is the state vector, $\boldsymbol{z}_{k} \in \mathbb{R}^{m}$ is the measurement vector and $\boldsymbol{w}(t) \in \mathbb{R}^{p}, \boldsymbol{v}_{k} \in \mathbb{R}^{s}$ are the process and measurement noise vectors, respectively. The matrices $\mathbf{A}, \mathbf{B}, \mathbf{C}_{k}$ and $\mathbf{D}_{k}$ are known and of appropriate dimension. Many systems encountered in practice are described by Eq. (1). State dynamics are often derived from physical laws (e.g., Newton's laws of motion) and take the form of continuous-time differential equations, whereas external measurements of a system are usually taken with digital sensors that provide data at discrete-time epochs.

The components of $\boldsymbol{w}$ and $\boldsymbol{v}_{k}$ are known to be the sum of a first order Gauss-Markov process and zero-mean white Gaussian noise. That is

$$
\begin{aligned}
\boldsymbol{w}(t) & =\mathbf{E}_{w} \boldsymbol{a}(t)+\boldsymbol{n}_{w}(t) & , & E\left[\boldsymbol{n}_{w}(t) \boldsymbol{n}_{w}^{T}(s)\right]=\mathbf{N}(t) \delta(t-s) \\
\boldsymbol{v}_{k} & =\mathbf{E}_{v} \boldsymbol{a}_{k}+\boldsymbol{n}_{v, k} & , & E\left[\boldsymbol{n}_{v, k} \boldsymbol{n}_{v, l}^{T}\right]=\mathbf{R}_{k} \delta_{k l} \\
\dot{\boldsymbol{a}} & =\mathbf{L} \boldsymbol{a}+\boldsymbol{u}(t) & , & E\left[\boldsymbol{u}(t) \boldsymbol{u}^{T}(s)\right]=\mathbf{U} \delta(t-s)
\end{aligned}
$$

such that $\mathbf{E}_{w}=\left[\begin{array}{ll}\mathbf{I}_{p} & \mathbf{0}_{p \times s}\end{array}\right], \mathbf{E}_{v}=\left[\begin{array}{ll}\mathbf{0}_{s \times p} & \mathbf{I}_{s}\end{array}\right], \delta(t)$ is the Dirac delta function, and $\delta_{k l}$ is the Kronecker delta. The power spectral density matrix $\mathbf{N}(t) \in \mathbb{R}^{p \times p}$ and covariance matrix $\mathbf{R}_{k} \in$ $\mathbb{R}^{S \times s}$ are

$$
\mathbf{N}(t)=\left[\begin{array}{lll}
n_{1}(t) & & \\
& \ddots & \\
& & n_{p}(t)
\end{array}\right] \text { and } \mathbf{R}_{k}=\left[\begin{array}{lll}
r_{1, k} & & \\
& \ddots & \\
& & r_{s, k}
\end{array}\right]
$$

For the Gauss-Markov state vector $\boldsymbol{a} \in \mathbb{R}^{p+s}, \mathbf{L}$ and $\mathbf{U}$ are [9]

$$
\mathbf{L}=\left[\begin{array}{ccc}
-\frac{1}{\tau_{1}} & & \\
& \ddots & \\
& & -\frac{1}{\tau_{p+s}}
\end{array}\right] \text { and } \mathbf{U}=\left[\begin{array}{ccc}
\frac{2 \sigma_{1}^{2}}{\tau_{1}} & & \\
& \ddots & \\
& & \frac{2 \sigma_{p+s}^{2}}{\tau_{p+s}}
\end{array}\right]
$$

The parameters $n_{i}(t), r_{j, k}, \sigma_{l}^{2}$ and $\tau_{l}$ are only known to reside in specified intervals.

\section{State Augmentation}

State augmentation [10] is a common technique to account for correlated noise and involves appending $\boldsymbol{a}$ to $\boldsymbol{\xi}$ to form the new linear system 


$$
\begin{aligned}
{\left[\begin{array}{c}
\dot{\xi} \\
\dot{\boldsymbol{a}}
\end{array}\right] } & =\left[\begin{array}{cc}
\mathbf{A}(t) & \mathbf{B}(t) \mathbf{E}_{w} \\
\mathbf{0} & \mathbf{L}
\end{array}\right]\left[\begin{array}{l}
\xi \\
\boldsymbol{a}
\end{array}\right]+\left[\begin{array}{c}
\mathbf{B}(t) \boldsymbol{n}_{w}(t) \\
\boldsymbol{u}(t)
\end{array}\right] \\
\boldsymbol{z}_{k} & =\left[\begin{array}{ll}
\mathbf{C}_{k} & \mathbf{D}_{k} \mathbf{E}_{v}
\end{array}\right]\left[\begin{array}{l}
\xi_{k} \\
\boldsymbol{a}_{k}
\end{array}\right]+\mathbf{D}_{k} \boldsymbol{n}_{v, k}
\end{aligned}
$$

In more compact notation,

$$
\begin{aligned}
\dot{\boldsymbol{x}} & =\mathbf{F}(t) \boldsymbol{x}+\boldsymbol{q}(t) \\
\boldsymbol{z}_{k} & =\mathbf{H}_{k} \boldsymbol{x}_{k}+\mathbf{D}_{k} \boldsymbol{n}_{v, k}
\end{aligned}
$$

Equation (6) is a linear system driven by zero-mean white Gaussian noise, thus it is permissible to estimate the augmented state vector $\boldsymbol{x}$ using a KF.

Since $n_{i}(t), r_{j, k}, \sigma_{l}^{2}$ and $\tau_{l}$ are unknown, it is not clear what values should be used to define the $\mathrm{KF}$. One criterion is to use parameter values so that the predicted estimate error distribution overbounds the true error distribution over the range of admissible values. To define an overbounding distribution, let $\hat{y}$ be an estimate of a specified state $y$ and define $\varepsilon_{y}=y-\hat{y}$ as the associated estimate error. Then for a maximum tolerable error $\ell_{y}$, an overbounding distribution is one that produces an upper bound on the integrity risk $P\left(\left|\varepsilon_{y}\right|>\ell_{y}\right)$.

Given that Eq. (6) is a linear system driven by zero-mean Gaussian noise and the fact that the KF is a linear unbiased estimator, it follows that $\varepsilon_{y} \sim N\left(0, \sigma_{y}^{2}\right)$. In this case, an upper bound on integrity risk is equivalent to an upper bound on the estimate error variance $\sigma_{y}^{2}$. Therefore, subsequent sections of the paper will focus on developing an upper bound on the estimate error variance for any state of interest.

\section{Motivational Example}

Before developing a rigorous approach for defining an overbounding distribution in the presence of uncertainty, it is instructive to address the fallacies in current thinking. The conventional wisdom is that a KF using the upper bound parameter values will produce an estimate error covariance matrix that overbounds the true error distribution. We'll use a simple one-dimensional estimation problem to demonstrate that this heuristic is not always accurate.

Consider the vehicle in Figure 1 moving at constant speed $u$ along the $x$ axis from an initial point $p_{0}$.

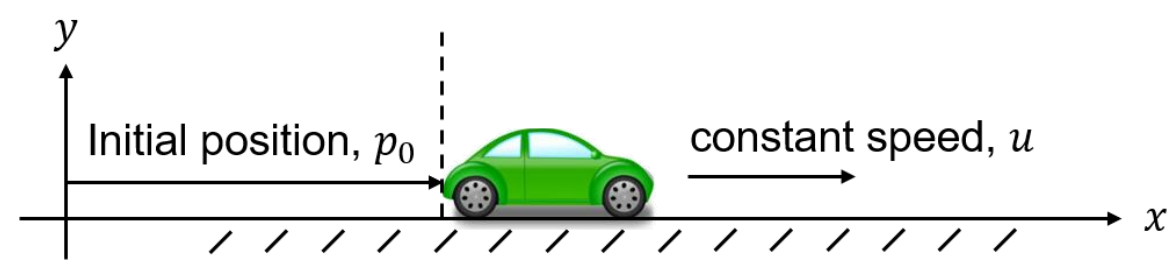

Figure 1. One-dimensional position and velocity estimation. 
Noisy measurements of the current position are available, described by the model

$$
z_{k}=\left[\begin{array}{ll}
1 & k \Delta t
\end{array}\right]\left[\begin{array}{c}
p_{0} \\
u
\end{array}\right]+v_{k}
$$

where $k$ is a time index, $\Delta t$ is the time interval between measurements and $v_{k}$ is the sum of a first order Gauss-Markov process and zero-mean white Gaussian noise. That is

$$
\begin{aligned}
v_{k} & =a_{k}+n_{k} \quad, \quad n_{k} \sim N\left(0, \sigma_{n}^{2}\right) \\
a_{k+1} & =e^{-\Delta t / \tau} w_{k}, \quad w_{k} \sim N\left(0, \sigma_{a}^{2}\left(1-e^{-2 \Delta t / \tau}\right)\right)
\end{aligned}
$$

The variances are known and given by $\sigma_{n}^{2}=0.5 \mathrm{~m}^{2}$ and $\sigma_{a}^{2}=1 \mathrm{~m}^{2}$. However, the time constant $\tau$ is only known to lie in the interval $[10,100] \mathrm{s}$. Noting that $p_{0}$ and $u$ are constants, state augmentation results in the estimation problem

$$
\begin{aligned}
{\left[\begin{array}{c}
p_{0, k+1} \\
u_{k+1} \\
a_{k+1}
\end{array}\right] } & =\left[\begin{array}{ccc}
1 & 0 & 0 \\
0 & 1 & 0 \\
0 & 0 & e^{-\Delta t / \tau}
\end{array}\right]\left[\begin{array}{c}
p_{0, k} \\
u_{k} \\
a_{k}
\end{array}\right]+\left[\begin{array}{c}
0 \\
0 \\
w_{k}
\end{array}\right] \\
z_{k} & =\left[\begin{array}{lll}
1 & k \Delta t & 1
\end{array}\right]\left[\begin{array}{c}
p_{0, k} \\
u_{k} \\
a_{k}
\end{array}\right]+n_{k}
\end{aligned}
$$

with initial covariance matrix $\mathbf{P}_{0}=\operatorname{diag}\left(100 \mathrm{~m}^{2}, 1 \mathrm{~m}^{2} / \mathrm{s}^{2}, 1 \mathrm{~m}^{2}\right)$.

One approach to address the uncertainty in $\tau$ is to run a bank of KFs, with each filter hypothesizing a different value for $\tau$. The estimate error variance at a given point in time would then be defined as the maximum variance over all filters in the bank. This approach is highly inefficient from a practical perspective, unless one value of $\tau$ (we think $\tau=\tau_{\max }$ ) happens to produce the maximum variance at all time instances. Figure 2 shows the results from running a two-filter bank.

For the initial position and speed states, $\tau_{\max }$ clearly does not produce an upper bound on the estimate error variance at all time instances. It is also interesting to note that the cross-over point between the two filters is state-dependent, thus any insight that may be gained into the behavior of one state does not necessarily translate to other filter states. The conclusions from this simple example are: 1) the intuitive notion that $\tau_{\max }$ is the worst-case time constant is not always true and 2) a more rigorous approach is needed to establish an overbounding estimate error distribution when correlated measurement error models are uncertain. 

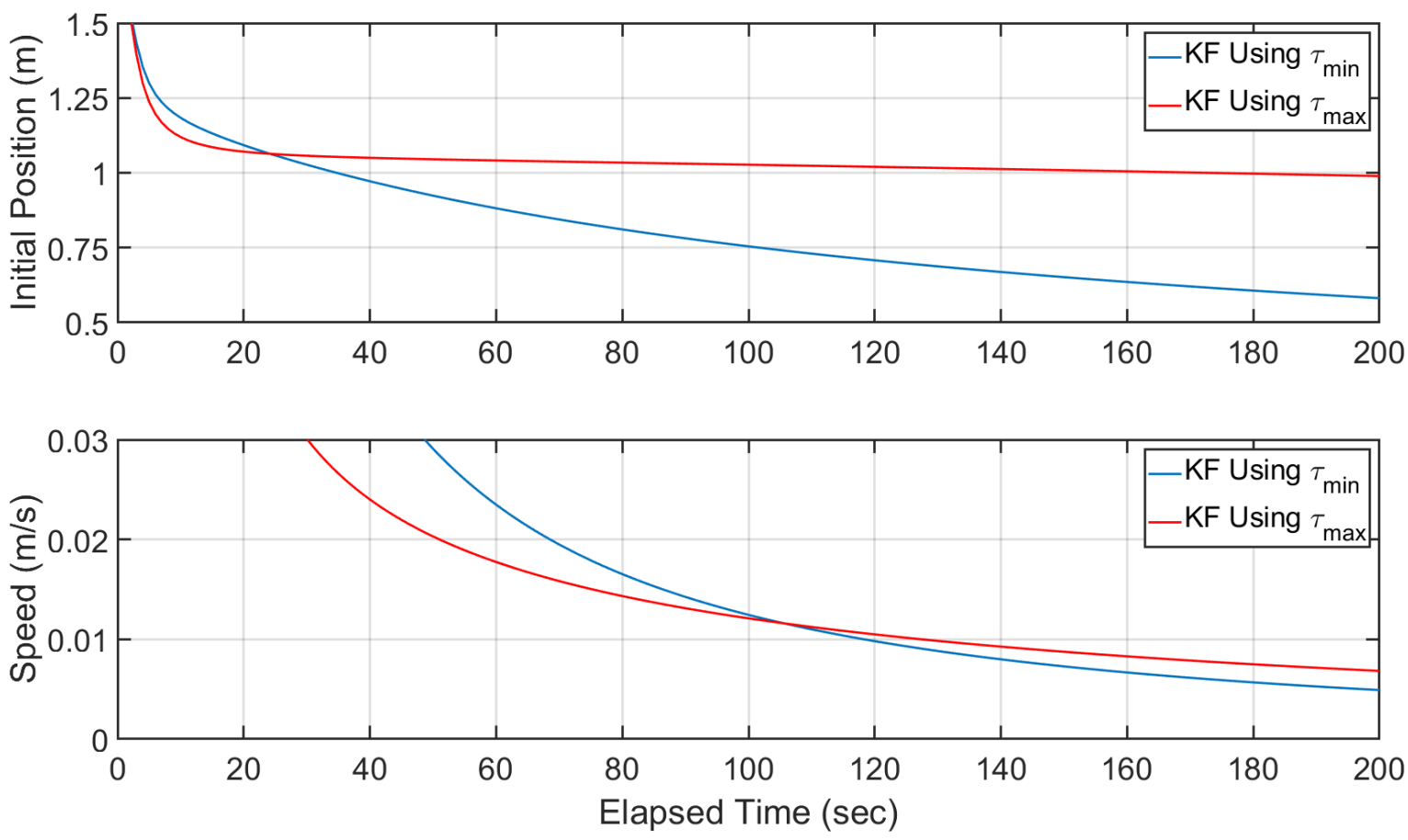

Figure 2. Predicted estimate error standard deviations using two different values for $\tau$.

\section{True Covariance Matrix}

The notation $(\cdot)_{k \mid k}$ will be used to denote a quantity at time index $k$ based on all measurements up to and including time index $k$. Occasionally the subscript $k \mid k$ will be dropped to denote a quantity at an arbitrary time. The traditional KF estimate error equations do not capture the effect of uncertain parameters and hence do not accurately describe the true estimate error statistics when $n_{i}(t), r_{j, k}, \sigma_{l}^{2}$ and $\tau_{l}$ are unknown but bounded. In response, new equations must be derived to propagate the estimate error vector.

We adopt the approach taken in Ref. [9], where generalized equations are derived to propagate $\boldsymbol{e}^{T}=\left[\begin{array}{ll}\boldsymbol{\varepsilon}^{T} & \boldsymbol{a}^{T}\end{array}\right]$ such that $\boldsymbol{\varepsilon}=\boldsymbol{x}-\widehat{\boldsymbol{x}}$. These equations allow us to determine the true covariance matrix $\mathbf{P}=E\left[\boldsymbol{e} \boldsymbol{e}^{T}\right]$. The KF propagates the estimate vector $\widehat{\boldsymbol{x}}$ according to

$$
\begin{aligned}
& \dot{\hat{\boldsymbol{x}}}=\hat{\mathbf{F}}(t) \widehat{\boldsymbol{x}}, t \in\left[t_{k-1}, t_{k}\right] \\
& \widehat{\boldsymbol{x}}\left(t_{k-1}\right)=\widehat{\boldsymbol{x}}_{k-1 \mid k-1} \\
& \widehat{\boldsymbol{x}}_{k \mid k}=\widehat{\boldsymbol{x}}_{k \mid k-1}+\mathbf{K}_{k}\left(\mathbf{z}_{k}-\mathbf{H}_{k} \widehat{\boldsymbol{x}}_{k \mid k-1}\right)
\end{aligned}
$$

where the notation $\hat{\mathbf{F}}(t)$ accentuates the fact that the KF is using a dynamic model $\hat{\mathbf{L}}$ for the GaussMarkov states that in general is not equal to the true dynamic model, $\mathbf{L}$. With $\Delta \mathbf{F}^{T}=\left[\begin{array}{ll}\mathbf{0} & \Delta \mathbf{L}^{T}\end{array}\right]$, where $\Delta \mathbf{L}=\mathbf{L}-\hat{\mathbf{L}}$, Appendix A shows that $\mathbf{P}$ propagates according to 


$$
\begin{aligned}
& \dot{\mathbf{P}}=\left[\begin{array}{cc}
\hat{\mathbf{F}}(t) & \Delta \mathbf{F} \\
\mathbf{0} & \mathbf{L}
\end{array}\right] \mathbf{P}+\mathbf{P}\left[\begin{array}{cc}
\hat{\mathbf{F}}^{T}(t) & \mathbf{0} \\
\Delta \mathbf{F}^{T} & \mathbf{L}^{T}
\end{array}\right]+\mathbf{Q}(t), t \in\left[t_{k-1}, t_{k}\right] \\
& \mathbf{P}\left(t_{k-1}\right)=\mathbf{P}_{k-1 \mid k-1} \\
& \mathbf{P}_{k \mid k}=\left[\begin{array}{cc}
\mathbf{I}-\mathbf{K}_{k} \mathbf{H}_{k} & \mathbf{0} \\
\mathbf{0} & \mathbf{I}
\end{array}\right] \mathbf{P}_{k \mid k-1}\left[\begin{array}{cc}
\left(\mathbf{I}-\mathbf{K}_{k} \mathbf{H}_{k}\right)^{T} & \mathbf{0} \\
\mathbf{0} & \mathbf{I}
\end{array}\right]+\left[\begin{array}{c}
\mathbf{K}_{k} \mathbf{D}_{k} \mathbf{R}_{k} \mathbf{D}_{k}^{T} \mathbf{K}_{k}^{T} \\
\mathbf{0}
\end{array}\right]
\end{aligned}
$$

such that

$$
\mathbf{Q}(t)=\left[\begin{array}{ccc}
\mathbf{B}(t) \mathbf{N}(t) \mathbf{B}^{T}(t) & \mathbf{0} & \mathbf{0} \\
\mathbf{0} & \mathbf{U} & \mathbf{U} \\
\mathbf{0} & \mathbf{U} & \mathbf{U}
\end{array}\right]
$$

Equations (11) and (12) properly reflect the fact that $n_{i}(t), r_{j, k}, \sigma_{l}^{2}$ and $\tau_{l}$ are uncertain. However, $\mathbf{P}$ cannot be computed in practice because $\Delta \mathbf{F}$ is unknown. Instead, we seek to define a covariance matrix $\boldsymbol{\Sigma}$ such that $(\boldsymbol{\Sigma}-\mathbf{P})>0$ (i.e., $\boldsymbol{\Sigma}-\mathbf{P}$ is positive semidefinite). For a specified state of interest $y=\boldsymbol{\beta}^{T} \boldsymbol{e}$, the condition $(\boldsymbol{\Sigma}-\mathbf{P})>0$ ensures that $\boldsymbol{\beta}^{T}(\boldsymbol{\Sigma}-\mathbf{P}) \boldsymbol{\beta}>0$. That is, the predicted variance $\boldsymbol{\beta}^{T} \boldsymbol{\Sigma} \boldsymbol{\beta}$ is an upper bound on the true variance $\boldsymbol{\beta}^{T} \mathbf{P} \boldsymbol{\beta}$ and thus leads to an overbounding distribution.

\section{Bounding Covariance Matrix}

As a starting point, let $\boldsymbol{\Sigma}$ be defined by the update equations

$$
\begin{gathered}
\dot{\boldsymbol{\Sigma}}=\left[\begin{array}{cc}
\hat{\mathbf{F}}(t) & \Delta \mathbf{F} \\
\mathbf{0} & \mathbf{L}
\end{array}\right] \boldsymbol{\Sigma}+\boldsymbol{\Sigma}\left[\begin{array}{cc}
\hat{\mathbf{F}}^{T}(t) & \mathbf{0} \\
\Delta \mathbf{F}^{T} & \mathbf{L}^{T}
\end{array}\right]+\widehat{\mathbf{Q}}(t)+\left[\begin{array}{ll}
\mathbf{0} & \mathbf{0} \\
\mathbf{0} & \mathbf{S}
\end{array}\right], \\
\boldsymbol{\Sigma}\left(t_{k-1}\right)=\boldsymbol{\Sigma}_{k-1 \mid k-1} \\
\boldsymbol{\Sigma}_{k \mid k}=\left[\begin{array}{cc}
\mathbf{I}-\mathbf{K}_{k} \mathbf{H}_{k} & \mathbf{0} \\
\mathbf{0} & \mathbf{I}
\end{array}\right] \boldsymbol{\Sigma}_{k \mid k-1}\left[\begin{array}{cc}
\left(\mathbf{I}-\mathbf{K}_{k} \mathbf{H}_{k}\right)^{T} & \mathbf{0} \\
\mathbf{0} & \mathbf{I}
\end{array}\right]+\left[\begin{array}{c}
\mathbf{K}_{k} \mathbf{D}_{k} \widehat{\mathbf{R}}_{k} \mathbf{D}_{k}^{T} \mathbf{K}_{k}^{T} \\
\mathbf{0}
\end{array}\right]
\end{gathered}
$$

such that $\mathbf{S}>0, \widehat{\mathbf{Q}}(t) \geq 0$ and $\widehat{\mathbf{R}}_{k} \geq 0$ are matrices to be specified. The motivation for adding $\mathbf{S}$ is currently unclear, but we'll see later that it plays a critical role in determining the transition matrix $\hat{\mathbf{F}}(t)$ that guarantees $\boldsymbol{\Sigma} \geq \mathbf{P}$. The error matrix $\mathbf{E}=\boldsymbol{\Sigma}-\mathbf{P}$ propagates according to

$$
\begin{gathered}
\dot{\mathbf{E}}=\left[\begin{array}{cc}
\widehat{\mathbf{F}}(t) & \Delta \mathbf{F} \\
\mathbf{0} & \mathbf{L}
\end{array}\right] \mathbf{E}+\mathbf{E}\left[\begin{array}{cc}
\hat{\mathbf{F}}^{T}(t) & \mathbf{0} \\
\Delta \mathbf{F}^{T} & \mathbf{L}^{T}
\end{array}\right]+(\widehat{\mathbf{Q}}-\mathbf{Q})+\left[\begin{array}{ll}
\mathbf{0} & \mathbf{0} \\
\mathbf{0} & \mathbf{S}
\end{array}\right] \\
\mathbf{E}_{k \mid k}=\left[\begin{array}{cc}
\mathbf{I}-\mathbf{K}_{k} \mathbf{H}_{k} & \mathbf{0} \\
\mathbf{0} & \mathbf{I}
\end{array}\right] \mathbf{E}_{k \mid k-1}\left[\begin{array}{cc}
\mathbf{I}-\mathbf{K}_{k} \mathbf{H}_{k} & \mathbf{0} \\
\mathbf{0} & \mathbf{I}
\end{array}\right]^{T}+\left[\begin{array}{c}
\mathbf{K}_{k} \mathbf{D}_{k}\left(\widehat{\mathbf{R}}_{k}-\mathbf{R}_{k}\right) \mathbf{D}_{k}^{T} \mathbf{K}_{k}^{T} \\
\mathbf{0}
\end{array}\right]
\end{gathered}
$$

Equation (14a) is a Lyapunov matrix differential equation. It is well known that if the initial condition $\mathbf{E}(0) \geq 0$ and $\widehat{\mathbf{Q}} \geq \mathbf{Q}$, there exists a positive semi-definite solution $\mathbf{E}(t)$ for $t \geq 0$ (see [12]). Furthermore, if $\mathbf{E}(t) \geq 0$ and $\widehat{\mathbf{R}}_{k} \geq \mathbf{R}_{k}$, then the transformation in Eq. (14b) also ensures 
that $\mathbf{E}_{k \mid k} \geq 0$. The condition $\widehat{\mathbf{R}}_{k} \geq \mathbf{R}_{k}$ is achieved by defining $\widehat{\mathbf{R}}_{k}$ as the matrix $\mathbf{R}_{k}$ populated with the upper bound values for $r_{j, k}$.

To determine $\widehat{\mathbf{Q}}$, let $\widehat{\mathbf{U}}$ be the matrix $\mathbf{U}$ formed with the upper bound values for $\sigma_{l}^{2}$ and the lower bound values for $\tau_{l}$. Also define $\widehat{\mathbf{N}}(t)$ as the matrix $\mathbf{N}(t)$ populated with the upper bound values for $n_{i}(t)$. Then with $\widehat{\mathbf{Q}}$ defined as

$$
\widehat{\mathbf{Q}}=\left[\begin{array}{ccc}
\mathbf{B}(t) \widehat{\mathbf{N}}(t) \mathbf{B}^{T}(t) & \mathbf{0} & \mathbf{0} \\
\mathbf{0} & \widehat{\mathbf{U}} & \mathbf{U} \\
\mathbf{0} & \mathbf{U} & \mathbf{U}
\end{array}\right]
$$

it is clear that $(\widehat{\mathbf{Q}}-\mathbf{Q}) \geq 0$ for all admissible values of $n_{i}(t), \sigma_{l}^{2}$ and $\tau_{l}$.

Thus far, we've shown that upper bound values for $r_{j, k}, n_{i}(t)$ and $\sigma_{l}^{2}$ should be used to ensure that $\boldsymbol{\Sigma} \geq \mathbf{P}$. This result is comforting because it appeals to the intuition that larger measurement noise variances (and power spectral densities) translate to a larger estimate error variance. It was also shown that the lower bound for the time constants $\tau_{l}$ should be used in the process noise power spectral density matrix $\widehat{\mathbf{Q}}$. The next step is to address the unknown matrix $\Delta \mathbf{F}$ in Eq. (14) and specify the transition matrix $\hat{\mathbf{F}}(t)$.

\section{Establishing the Transition Matrix}

After forming the partitioned matrix $\boldsymbol{\Sigma}=\left[\begin{array}{cc}\boldsymbol{\Sigma}_{x} & \boldsymbol{\Sigma}_{x a} \\ \boldsymbol{\Sigma}_{x a}^{T} & \boldsymbol{\Sigma}_{a}\end{array}\right]$, the expanded version of Eq. (13a) is

$$
\begin{aligned}
\dot{\boldsymbol{\Sigma}}_{x} & =\hat{\mathbf{F}}(t) \boldsymbol{\Sigma}_{x}+\Delta \mathbf{F} \boldsymbol{\Sigma}_{x a}^{T}+\boldsymbol{\Sigma}_{x} \hat{\mathbf{F}}^{T}(t)+\boldsymbol{\Sigma}_{x a} \Delta \mathbf{F}^{T}+\widehat{\mathbf{Q}}_{x}(t) \\
\dot{\boldsymbol{\Sigma}}_{x a} & =\widehat{\mathbf{F}}(t) \boldsymbol{\Sigma}_{x a}+\Delta \mathbf{F} \boldsymbol{\Sigma}_{a}+\boldsymbol{\Sigma}_{x a} \mathbf{L}^{T}+\mathbf{Q}_{x a} \\
\dot{\boldsymbol{\Sigma}}_{a} & =\mathbf{L} \boldsymbol{\Sigma}_{a}+\boldsymbol{\Sigma}_{a} \mathbf{L}^{T}+\mathbf{U}+\mathbf{S}
\end{aligned}
$$

where

$$
\widehat{\mathbf{Q}}_{x}(t)=\left[\begin{array}{cc}
\mathbf{B}(t) \widehat{\mathbf{N}}(t) \mathbf{B}^{T}(t) & \mathbf{0} \\
\mathbf{0} & \widehat{\mathbf{U}}
\end{array}\right] \text { and } \mathbf{Q}_{x a}=\left[\begin{array}{l}
\mathbf{0} \\
\mathbf{U}
\end{array}\right]
$$

We only care about propagating $\boldsymbol{\Sigma}_{x}$, the block of $\boldsymbol{\Sigma}$ corresponding to $\boldsymbol{\varepsilon}$. In order to propagate $\boldsymbol{\Sigma}_{x}$, the unknown matrix $\Delta \mathbf{F}$ in Eq. (16a) must be eliminated. This is achieved by ensuring that $\boldsymbol{\Sigma}_{x a}(t)=\mathbf{0}$ for all $t$. Setting the initial condition $\boldsymbol{\Sigma}_{x a}(0)=\mathbf{0}$ and enforcing the constraint

$$
\Delta \mathbf{F} \boldsymbol{\Sigma}_{a}(t)+\mathbf{Q}_{x a}=\mathbf{0} \Rightarrow \Delta \mathbf{L} \boldsymbol{\Sigma}_{a}(t)+\mathbf{U}=\mathbf{0}
$$

transforms Eq. (16b) into the homogeneous differential equation $\dot{\boldsymbol{\Sigma}}_{x a}=\hat{\mathbf{F}}(t) \boldsymbol{\Sigma}_{x a}+\boldsymbol{\Sigma}_{x a} \mathbf{L}^{T}$, the solution to which is $\boldsymbol{\Sigma}_{x a}(t)=\mathbf{0}$, as desired. 
The Gauss-Markov process is a stationary process, i.e., $\dot{\boldsymbol{\Sigma}}_{a}=\mathbf{0}$. Therefore, Eq. (16c) becomes $\mathbf{L} \boldsymbol{\Sigma}_{a}+\boldsymbol{\Sigma}_{a} \mathbf{L}^{T}+\mathbf{U}+\mathbf{S}=\mathbf{0}$, which, after substituting $\mathbf{U}=(\hat{\mathbf{L}}-\mathbf{L}) \boldsymbol{\Sigma}_{a}$ from Eq. (18), simplifies to $\boldsymbol{\Sigma}_{a} \mathbf{L}^{T}+\hat{\mathbf{L}} \boldsymbol{\Sigma}_{a}+\mathbf{S}=\mathbf{0}$. Since $\mathbf{S}>0$, it must be that $\boldsymbol{\Sigma}_{a} \mathbf{L}^{T}+\hat{\mathbf{L}} \boldsymbol{\Sigma}_{a}<0$. This expression together with Eq. (18) define two conditions that must be satisfied in order to guarantee that $\boldsymbol{\Sigma}>\mathbf{P}$, namely

$$
\Delta \mathbf{L} \boldsymbol{\Sigma}_{a}(t)+\mathbf{U}=\mathbf{0} \text { and } \boldsymbol{\Sigma}_{a} \mathbf{L}^{T}+\hat{\mathbf{L}} \boldsymbol{\Sigma}_{a}<0
$$

All matrices in Eq. (19) are diagonal, leading (after algebraic manipulation) to the scalar conditions

$$
\begin{gathered}
\left(-1+\frac{\tau_{l}}{\hat{\tau}_{l}}\right)+\frac{2 \sigma_{l}^{2}}{\bar{\sigma}_{l}^{2}}=0 \\
\left(\frac{1}{\hat{\tau}_{l}}+\frac{1}{\tau_{l}}\right)>0
\end{gathered}
$$

where $\bar{\sigma}_{l}^{2}$ is the $l^{\text {th }}$ diagonal element of $\Sigma_{a}(t)$.

The Gauss-Markov process is only stable for positive time constants. Therefore, Eq. (20b) is always satisfied. Given that variances are nonnegative, the only way to satisfy Eq. (20a) for all admissible $\tau_{l}$ is to set $\hat{\tau}_{l}=\tau_{l, \max }$.

Substituting $\hat{\tau}_{l}=\tau_{l \text {,max }}$ into Eq. (20a) leads to the conclusion that

$$
\bar{\sigma}_{l}^{2}=\left(\frac{2}{1-\tau_{l} / \tau_{l, \text { max }}}\right) \sigma_{l}^{2}
$$

Equation (21) has important ramifications for initialization, which is covered in the next section.

\section{Discretization and Initialization}

Thus far we have shown that for first-order Gauss-Markov noise with unknown variance $\sigma^{2} \in$ $\left[\sigma_{\text {min }}^{2}, \sigma_{\text {max }}^{2}\right]$ and unknown time constant $\tau \in\left[\tau_{\min }, \tau_{\max }\right]$, the $\mathrm{KF}$ covariance matrix will overbound the estimate error distribution when the noise is modeled according to

$$
\dot{a}=-\frac{1}{\tau_{\max }} a+\sqrt{\frac{2 \sigma_{\max }^{2}}{\tau_{\min }}} w(t), \begin{aligned}
& w(t) \sim N(0,1) \\
& E[w(t) w(s)]=\delta(t-s)
\end{aligned}
$$

Appendix B shows that the corresponding discrete-time model is

$$
a_{k+1}=\exp \left(-\frac{\Delta t}{\tau_{\max }}\right) a_{k}+\sqrt{\sigma_{\max }^{2}\left(\frac{\tau_{\max }}{\tau_{\min }}\right)\left[1-\exp \left(-\frac{2 \Delta t}{\tau_{\max }}\right)\right]} w_{k}, \begin{aligned}
& w_{k} \sim N(0,1) \\
& E\left[w_{k} w_{l}\right]=\delta_{k l}
\end{aligned}
$$


The only remaining question is how to set the initial variance $\sigma_{a, 0}^{2}$. Recall from the discussion after Eq. (14b) that the condition $\mathbf{E}(0) \geq 0$ was a necessary condition to ensure that $\boldsymbol{\Sigma} \geq \mathbf{P}$ at all subsequent times. If $\boldsymbol{\varepsilon}_{\xi, 0}$ and $\boldsymbol{\varepsilon}_{a, 0}$ are initially uncorrelated, Appendix $\mathrm{C}$ shows that $\mathbf{E}(0) \geq 0$ implies that

$$
\sigma_{a, 0}^{2} \geq \frac{2 \sigma_{\max }^{2}}{\left(1+\tau_{\min } / \tau_{\max }\right)}
$$

Since there is no reason to make $\sigma_{a, 0}^{2}$ any larger than necessary, it is set equal to its lower bound in Eq. (24).

As a point of theoretical interest, notice that if $\sigma_{a, 0}^{2}$ were initialized with the larger variance $\sigma_{\max }^{2}\left(\tau_{\max } / \tau_{\min }\right)$, Eq. (23) would be a stationary Gauss-Markov process with time constant $\tau_{\max }$ and variance $\sigma_{\max }^{2}\left(\tau_{\max } / \tau_{\min }\right)$. This makes intuitive sense because we would be using a stationary model to conservatively account for uncertain Gauss-Markov noise. However, Eq. (24) shows that the least conservative model is a non-stationary model, even though the underlying noise processes are truly stationary.

\section{Summary of Theoretical Results}

To conclude the theoretical sections of the paper, we summarize the main result:

For first-order Gauss-Markov noise with an uncertain variance $\sigma^{2} \in\left[\sigma_{\min }^{2}, \sigma_{\max }^{2}\right]$ and uncertain time constant $\tau \in\left[\tau_{\min }, \tau_{\max }\right]$, the covariance matrix computed by a stateaugmented Kalman filter will overbound the estimate error distribution (in a positive semidefinite sense) when the filter uses the following model for the augmented states

$$
\begin{aligned}
a_{k+1} & =\exp \left(-\frac{\Delta t}{\tau_{\max }}\right) a_{k}+\sqrt{\sigma_{\max }^{2}\left(\frac{\tau_{\max }}{\tau_{\min }}\right)\left[1-\exp \left(-\frac{2 \Delta t}{\tau_{\max }}\right)\right]} w_{k}, \begin{array}{l}
w_{k} \sim N(0,1) \\
E\left[w_{k} w_{l}\right]=\delta_{k l}
\end{array} \\
\sigma_{a, 0}^{2} & =\frac{2 \sigma_{\max }^{2}}{\left(1+\tau_{\min } / \tau_{\max }\right)}
\end{aligned}
$$

\section{Example Application: Batch ARAIM Performance Analysis}

In this section, the estimation error bounding method described above is implemented in an example application of aircraft navigation using Advanced Receiver Autonomous Integrity Monitoring (ARAIM) [13, 14, 2]. The baseline ARAIM algorithm is a 'snapshot' implementation that uses dual-frequency multi-constellation measurements at one time-instant to achieve LPV200 requirements, i.e., requirements for localizer performance with vertical guidance down to 200 feet above the runway $[14,2]$.

However, when nominal GPS and Galileo constellations are depleted, LPV-200 can only be sparsely achieved using snapshot ARAIM [14]. 'Batch-ARAIM' addresses this limitation by 
processing measurements over time [3, 4, 15] using a sliding-window mechanism. One major challenge in batch ARAIM is to derive robust models of measurement errors over time. In prior work [4, 15], a 'bias-plus-ramp' model was derived for satellite clock and orbit error dynamics using nine months of data. In parallel, assumptions were made on the time correlation of tropospheric and multipath errors. Because no method was available to account for uncertainty in correlation time constants, the largest measured value was employed for multipath, and sensitivity to residual tropospheric delay was analyzed $[3,4,15]$. But multipath time constants can take a wide range of values as reported in Figures 21-22 of [5].

In this paper, we implement the new bounding method derived in the previous section to account for uncertainty in the correlation time constant of multipath and tropospheric errors in a batch ARAIM performance analysis. This section briefly describes the main assumptions of the multipath and troposphere error models, and then focuses on quantifying the impact of error correlation model uncertainty on ARAIM performance. Readers may refer to [3, 4, 15] for details on the batch ARAIM implementation. Also, while the above estimation error bounding method was derived for a KF, batch ARAIM uses a batch estimator. Keeping in mind that the current-time estimates of batch versus KF coincide, readers interested in the details of the KF versus batch implementations may refer to [16-18].

\section{Description of Batch ARAIM Assumptions}

We consider the following batch measurement equation:

$$
\boldsymbol{z}_{B, k}=\mathbf{C}_{B, k} \xi_{B, k}+\boldsymbol{v}_{B, k}
$$

where

$\boldsymbol{z}_{B, k} \quad$ : is the batch measurement vector comprised of raw (i.e., unfiltered) ionosphere-free code and carrier ranging measurements for all visible and healthy GPS and Galileo satellites during the batch interval, at all batch sampling times during the interval.

$\xi_{B, k} \quad$ : is the state vector composed of antenna positions and GPS/Galileo receiver clock biases at all batch sample times, time-invariant, float-valued carrier phase cycle ambiguities, and time-invariant clock and orbit error bias and ramp parameters for each satellite.

$\mathbf{C}_{B, k}$ : is the observation matrix including all state coefficients as described in $[3,4,15]$.

$\boldsymbol{v}_{B, k}$ : is the vector of all measurement error contributions, including residual orbit and clock errors, tropospheric delay, multipath and receiver noise. $\boldsymbol{v}_{B, k}$ is normally distributed with zero mean and covariance matrix $\boldsymbol{V}_{B, k}$.

Additional ARAIM error model parameters, such as the bounded bias accounting for non-Gaussian ranging errors [14, 2], are included in the simulation as explained in [15] but are not described here because they are not directly relevant to the proposed new bounding method.

Measurement error correlation is modeled in the off-diagonal components of the covariance matrix $\boldsymbol{V}_{B, k}$. First, code-carrier correlation due to common residual orbit and clock and tropospheric errors are accounted for as described in the appendix section of [15]. Then, off-diagonal components also account for the time-correlation due to multipath and tropospheric errors modeled as first order 
Gauss Markov Processes (GMP) [4, 15] with correlation time constants $\tau_{M}$ and $\tau_{T}$, respectively. The values of $\tau_{M}$ and $\tau_{T}$ are unknown but bounded following the inequalities:

$$
\begin{aligned}
\tau_{M, \min } & \leq \tau_{M} \leq \tau_{M, \max } \\
\tau_{T, \min } & \leq \tau_{T} \leq \tau_{T, \max }
\end{aligned}
$$

In prior work $[3,4,15]$, we used the maximum values $\tau_{M, \max }$ for the multipath correlation time constant and evaluated three different time-correlation models for the troposphere delay. The 'Motivational Example' Section of this paper shows that this is insufficient to overbound the estimate error distribution. In response, we use Eq. (27) in the batch ARAIM estimator and multiple hypothesis solution separation (MHSS) method derived in $[3,4,15]$. In order to evaluate batch ARAIM performance, we assume nominal error model parameter values given in [15]. A constant batch period of $10 \mathrm{~min}$ is assumed throughout the section. Other relevant parameter values for multipath and troposphere error models are given in Table I.

Table I. Relevant parameter values for multipath and troposphere error models (all other simulation parameters are given in [15])

\begin{tabular}{|l|c|c|}
\hline Parameter description & Symbol & Value \\
\hline Upper bound on the multipath error correlation time constant & $\tau_{M, \max }$ & $900 \mathrm{~s}$ \\
\hline Lower bound on the multipath error correlation time constant & $\tau_{M, \min }$ & $10 \mathrm{~s}$ \\
\hline $\begin{array}{l}\text { Variance of GMP used to model raw code multipath error at } \\
90 \text { deg elevation angle (model is elevation-dependent [2, 15]) }\end{array}$ & $\sigma_{M}^{2}$ & $(0.50 \mathrm{~m})^{2}$ \\
\hline Upper bound on the troposphere error correlation time constant & $\tau_{T, \max }$ & $900 \mathrm{~s}$ \\
\hline Lower bound on the troposphere error correlation time constant & $\tau_{T, \min }$ & $2700 \mathrm{~s}$ \\
\hline $\begin{array}{l}\text { Variance of GMP used to model zenith troposphere error } \\
\text { (model is elevation-dependent [2, 15]) }\end{array}$ & $\sigma_{T}^{2}$ & $(0.12 \mathrm{~m})^{2}$ \\
\hline
\end{tabular}

\section{Batch ARAIM Integrity Monitoring Performance Analysis}

In this subsection, we evaluate the increase in integrity risk bound caused by uncertainty in the measurement error correlation time constant. We then evaluate the impact of the new estimation error bounding method on batch-ARAIM as compared to snapshot ARAIM.

Nominal simulation parameters are defined in [15], and include:

- a five degree satellite elevation mask

- an integrity risk requirement of $10^{-7}$ per approach

- a continuity risk requirement of $4 \cdot 10^{-6}$ per approach

- a vertical alert limit, noted $\ell$, of $35 \mathrm{~m}$ (unless otherwise stated)

- a prior probability of satellite fault of $10^{-5}$

- a prior probability of constellation fault, noted $P_{\text {const }}$, of $10^{-4}$ (unless otherwise stated)

- a batch period of 10 minutes

- depleted constellations of '24-1' GPS satellites and '24-1' Galileo SVs [19] 
In Figure 3, the integrity risk is evaluated using batch MHSS [2, 15] over 24 hours, at an example Blacksburg, VA location (37.2 deg N, -80.4 deg E), assuming dual-frequency measurements from GPS and Galileo. In this figure, in order to accentuate differences between implementations, we use a vertical alert limit of $10 \mathrm{~m}$ (instead of $35 \mathrm{~m}$ ) and a prior probability of constellation faults of $10^{-8}$.

Figure 3 shows the significant integrity risk reduction obtained using batch ARAIM (thick curve and black circle markers) as compared to snapshot ARAIM (diamond markers). Figure 3 also shows that the new bound is more conservative than when simply assuming GMP correlation time constants of $\tau_{M, \max }$ and $\tau_{T, \max }$ for multipath and troposphere errors, respectively.

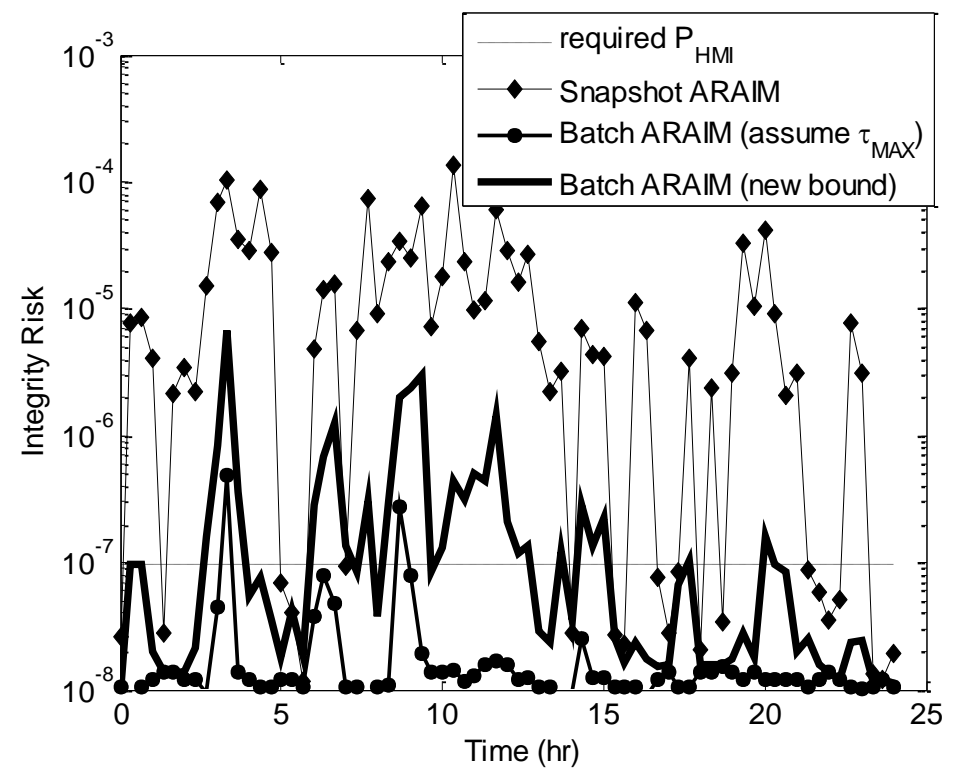

Figure 3. Integrity risk bound using snapshot versus batch ARAIM, with an optimistic assumption of $\tau=\tau_{\max }$ versus with the new bounding method, using depleted constellations, $P_{\text {const }}=10^{-8}, \ell$ $=10 \mathrm{~m}$.

In Figure 3, the fraction of time where the integrity risk curves are below the horizontal dotted line is the availability. In this case, availability is $91 \%$ for batch ARAIM when making the misleading and optimistic assumption of $\tau_{M}=\tau_{M, \max }$ and $\tau_{T}=\tau_{T, \max }$. It is expectedly lower, achieving $60 \%$, when accounting for our lack of knowledge on $\tau_{M}$ and $\tau_{T}$. But, using the new, more accurate model in batch ARAIM still provides a great availability improvement as compared to snapshot ARAIM, which does not exceed $26 \%$.

Figures 4 to 6 display global availability maps for a $10 \mathrm{deg} \times 10 \mathrm{deg}$ latitude-longitude grid of locations, for depleted '24-1' GPS and '24-1' Galileo constellations, for satellite geometries simulated at regular 10-minute intervals over a 24 hour period. We use nominal values of $\ell=35$ $\mathrm{m}$ and $P_{\text {const }}=10^{-4}$. Availability is computed at each location as the fraction of time where the integrity risk bound is lower than the $10^{-7}$ requirement. 

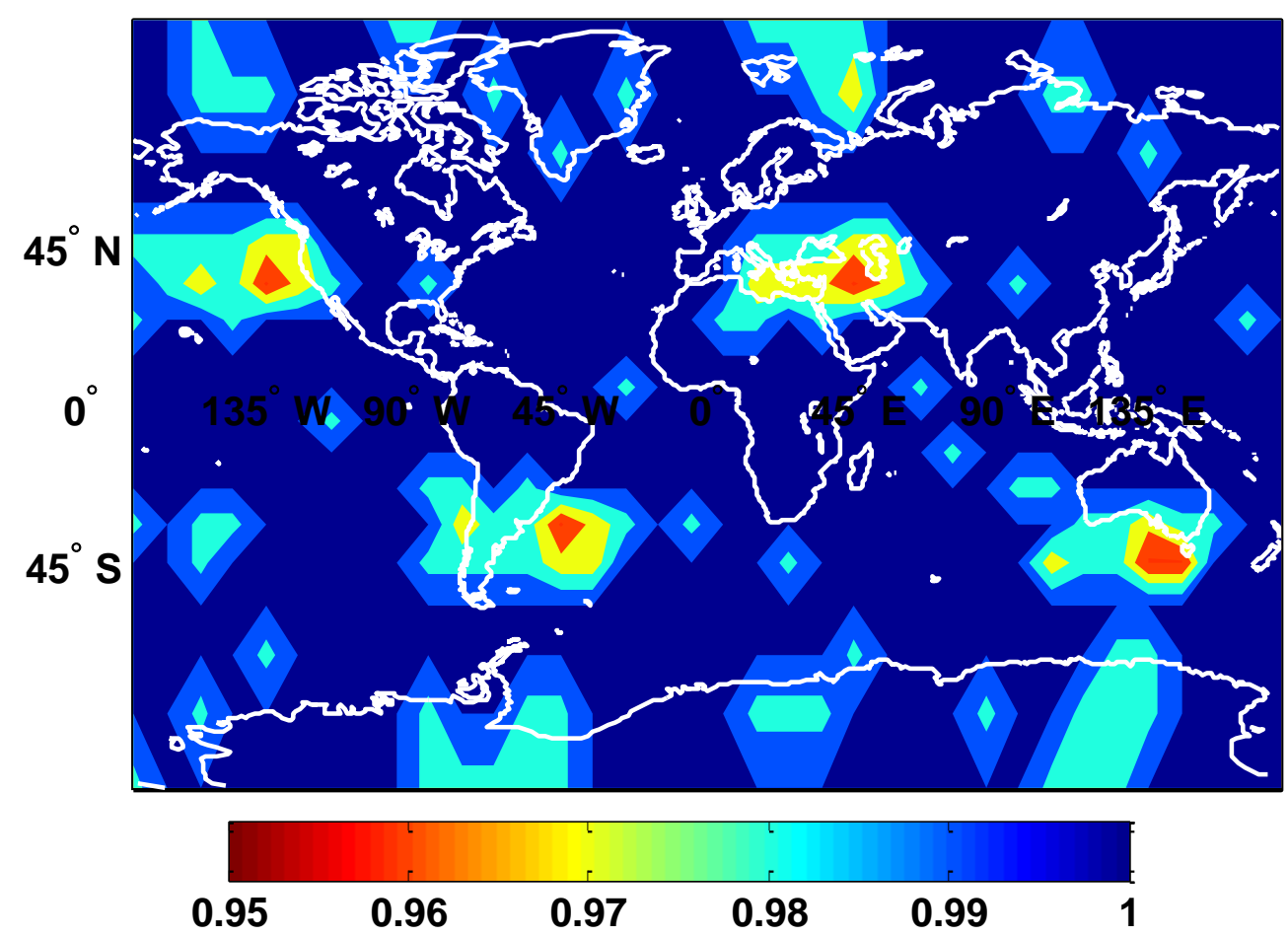

Figure 4. Availability map for snapshot ARAIM using depleted constellations, $P_{\text {const }}=10^{-4}, \ell=$ $35 \mathrm{~m}$ (coverage of $99.5 \%$ availability is $84.6 \%$ ).

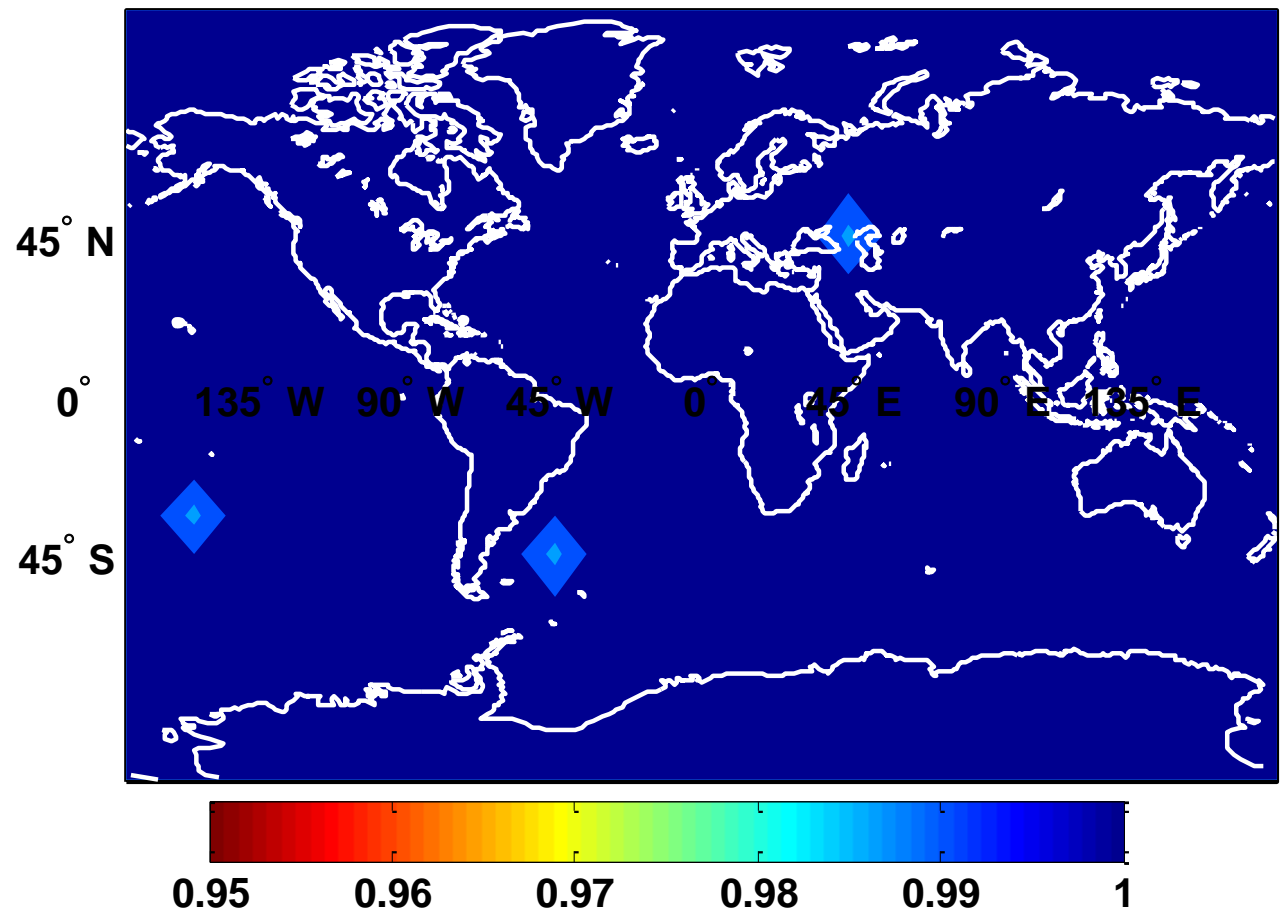

Figure 5. Availability for batch ARAIM using misleading, optimistic assumption $\tau=\tau_{\text {max }}$ using depleted constellations, $P_{\text {const }}=10^{-4}, \ell=35 \mathrm{~m}$ (coverage of $99.5 \%$ availability is $99.5 \%$ ). 


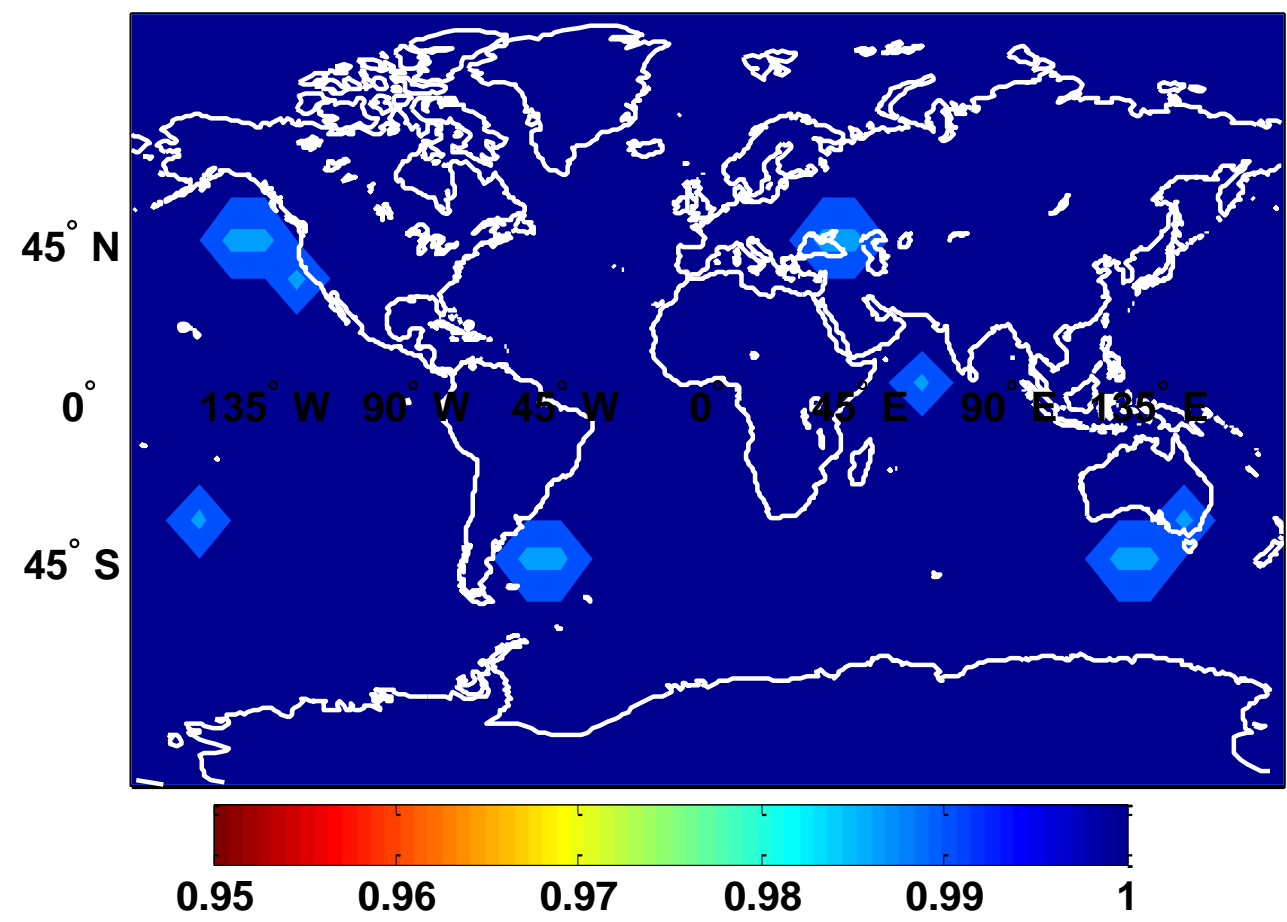

Figure 6. Availability map for batch ARAIM using the new bounding method using depleted constellations, $P_{\text {const }}=10^{-4}, \ell=35 \mathrm{~m}$ (coverage of $99.5 \%$ availability is $97.8 \%$ ).

The larger presence of blue areas in Figures 5 and 6 compared to Figure 4 clearly indicate that snapshot ARAIM is outperformed by batch ARAIM. Figure 5 makes the misleading, optimistic assumption $\tau_{M}=\tau_{M, \max }$ and $\tau_{T}=\tau_{T, \max }$. Figure 6 does not make that assumption and uses our new method instead, which accounts for measurement error time correlation uncertainty. Availability is still much higher in Figure 6 for batch ARAIM than in Figure 4 for snapshot ARAIM.

The worldwide availability metric given in the figure captions is the weighted coverage of $99.5 \%$ availability: coverage is defined as the percentage of grid point locations exceeding $99.5 \%$ availability. The coverage computation is weighted at each location by the cosine of the location's latitude, because grid point locations near the equator represent larger areas than near the poles. Table II lists worldwide coverage of $99.5 \%$ availability, and of $95 \%$ availability (given in parentheses). It shows that the coverage of $99.5 \%$ availability increases from $84.6 \%$ for snapshot ARAIM, to $99.5 \%$ for batch ARAIM assuming $\tau=\tau_{m a x}$, which we know is inaccurate. A more trustworthy number is provided by our new positioning and risk bounding method in Figure 6, which achieves $97.8 \%$ coverage, still more than $10 \%$ higher than snapshot ARAIM for the case considered here. These results would further improve if we had better knowledge of the measurement error sources, i.e., if we could reduce the difference between $\tau_{\max }$ and $\tau_{\text {min }}$. 
Table II. Coverage of $99.5 \%$ availability and coverage of $95 \%$ availability (in parentheses)

\begin{tabular}{|l|c|c|c|}
\hline & $\begin{array}{c}\text { Snapshot } \\
\text { ARAIM }\end{array}$ & $\begin{array}{c}\text { Batch ARAIM } \\
\text { assuming } \tau=\tau_{\max }\end{array}$ & $\begin{array}{c}\text { Batch ARAIM } \\
\text { new bounding method }\end{array}$ \\
\hline depleted constellations & $84.6 \%$ & $99.5 \%$ & $97.8 \%$ \\
$P_{\text {const }}=10^{-4}, \ell=35 \mathrm{~m}$ & $(100 \%)$ & $(100 \%)$ & $(100 \%)$ \\
\hline
\end{tabular}

\section{Conclusions}

A new approach was developed to obtain an overbounding distribution on KF estimate error when measurement and process noise are first-order Gauss Markov with an unknown variance and time constant. Specifically, it was shown that when Gauss Markov noise is modeled using $\tau_{\max }$ in the transition matrix and a process noise variance inflated by $\left(\tau_{\max } / \tau_{\min }\right)$, the $\mathrm{KF}$ covariance matrix is guaranteed to overbound the estimate error distribution. The minimum allowable initial variance was also derived, resulting in the tightest possible overbound. For an aircraft navigation application using batch ARAIM, it was shown that under specific circumstances, the new method produces a $10 \%$ improvement in $99.5 \%$ availability coverage compared to conventional snapshot RAIM. Future work will focus on extending the ideas presented in this paper beyond first order GaussMarkov noise, with the goal of developing practical overbounding methods that do not rely on precise mathematical knowledge of the underlying time correlation.

\section{References}

[1] Tupysev, V. A., Stepanov, O. A., Loparev, A. V., and Litvinenko, Y. A. "Guaranteed Estimation in the Problems of Navigation Information Processing." Proceedings of the $18^{\text {th }}$ IEEE International Conference on Control Applications, St. Petersburg, Russia, 2009, pp. $1672-1677$.

[2] Working Group C. “ARAIM Technical Subgroup. Milestone 3.0 Report." Technical report, EU-US Cooperation on Satellite Navigation, 2016.

[3] Joerger, M., and B. Pervan. "Multi-Constellation ARAIM Exploiting Satellite Geometry Change." Proceedings of the 28th International Technical Meeting of The Satellite Division of the Institute of Navigation (ION GNSS+ 2015), Tampa, FL, 2015.

[4] Joerger, Mathieu, Pervan, Boris, "Exploiting Satellite Motion in ARAIM: Measurement Error Model Refinement Using Experimental Data," Proceedings of the 29th International Technical Meeting of The Satellite Division of the Institute of Navigation (ION GNSS+ 2016), Portland, OR, 2016, pp. 1696-1712.

[5] Pervan, B., Khanafseh, S., and Patel, J. "Test Statistic Auto- and Cross-correlation Effects on Monitor False Alert and Missed Detection Probabilities," Proceedings of the 2017 International Technical Meeting of The Institute of Navigation, Monterey, California, 2017, pp. 562-590.

[6] Rife, J., and D. Gebre-Egziabher. "Symmetric Overbounding of Correlated Errors." NAVIGATION 54.2 (2007): 109-124.

[7] Pulford, G. W. "A Proof of the Spherically Symmetric Overbounding Theorem for Linear Systems." NAVIGATION 55.4 (2008): 283-292. 
[8] Langel, S., S. Khanafseh and B. Pervan. "Bounding Integrity Risk for Sequential State Estimators with Stochastic Modeling Uncertainty." AIAA J. Guidance, Control, and Dynamics 37.1 (2014): 36-46.

[9] Gelb, A. (ed.), Applied Optimal Estimation, The MIT Press, Cambridge, MA, 1974.

[10] Anderson, B. D. O., and Moore, J. B., Optimal Filtering, Dover Publications, Mineola, NY, 1979, Chap. 11, pp. 296-303.

[11] Simon, D., Optimal State Estimation: Kalman, H Infinity and Nonlinear Approaches, John Wiley \& Sons, Inc., Hoboken, NJ, 2006, Chap. 13, pp. 403.

[12] Dieci, L., Eirola, T., "Positive Definiteness in the Solution of Riccati Differential Equations." Numerische Mathematik 67.3 (1994): 303-313.

[13] Working Group C. “ARAIM Technical Subgroup. Interim Report Issue 1.0.” Technical report, EU-US Cooperation on Satellite Navigation, 2012.

[14] Working Group C. “ARAIM Technical Subgroup. Milestone 2.0 Report.” Technical report, EU-US Cooperation on Satellite Navigation, 2015.

[15] Joerger, M., and B. Pervan. "Multi-Constellation ARAIM Exploiting Satellite Geometry Change." in review. NAVIGATION. 2019.

[16] Joerger, M., Pervan, B., "Kalman Filter-Based Integrity Monitoring Against Sensor Faults." AIAA Journal of Guidance, Control and Dynamics, Vol. 36, No. 2, 2013, pp. 349-361.

[17] Joerger, M., "Carrier Phase GPS Augmentation Using Laser Scanners and Using Low Earth Orbiting Satellites." Ph.D. Dissertation, Illinois Institute of Technology, 2009.

[18] Crassidis, J., and Junkins, J., Optimal Estimation of Dynamic Systems, Chapman \& Hall/CRC, Boca Raton, FL, 2004, pp. 123-174.

[19] almanac files provided by Stanford University, available online at http://waas.stanford.edu/staff/maast/maast.html

[20] Brown, R. G., and Hwang, P. Y. C., Introduction to Random Signals and Applied Kalman Filtering, 4th ed., John Wiley \& Sons, Hoboken, NJ, 2012, pp. 126.

[21] Gallier, J., "The Schur Complement and Symmetric Positive Semidefinite (and Definite) Matrices," available online at https://www.cis.upenn.edu/ jean/schur-comp.pdf, 2019.

\section{Appendix A: True Estimate Error Covariance Matrix}

This appendix derives the true estimate error covariance for a state-augmented KF when there are uncertain parameters in the augmented state dynamic models. With $\Delta \mathbf{L}=\mathbf{L}-\hat{\mathbf{L}}$, the transition matrix in Eq. (5) can be written as

$$
\mathbf{F}(t)=\left[\begin{array}{cc}
\mathbf{A}(t) & \mathbf{B}(t) \mathbf{E}_{w} \\
\mathbf{0} & \hat{\mathbf{L}}+\Delta \mathbf{L}
\end{array}\right]=\hat{\mathbf{F}}(t)+\left[\begin{array}{cc}
\mathbf{0} & \mathbf{0} \\
\mathbf{0} & \Delta \mathbf{L}
\end{array}\right]
$$

Then the dynamic model $\dot{\boldsymbol{x}}=\mathbf{F}(t) \boldsymbol{x}+\boldsymbol{q}(t)$ in Eq. (6) becomes $\dot{\boldsymbol{x}}=\hat{\mathbf{F}}(t) \boldsymbol{x}+\left[\begin{array}{c}\mathbf{0} \\ \Delta \mathbf{L}\end{array}\right] \boldsymbol{a}(t)+\boldsymbol{q}(t)$. 
Noting that the KF estimate vector propagates as $\dot{\hat{\boldsymbol{x}}}=\widehat{\mathbf{F}}(t) \widehat{\boldsymbol{x}}$, the estimate error vector $\boldsymbol{\varepsilon}=\boldsymbol{x}-\widehat{\boldsymbol{x}}$ satisfies the differential equation

$$
\dot{\boldsymbol{\varepsilon}}=\hat{\mathbf{F}}(t) \boldsymbol{\varepsilon}+\left[\begin{array}{c}
\mathbf{0} \\
\Delta \mathbf{L}
\end{array}\right] \boldsymbol{a}(t)+\boldsymbol{q}(t)
$$

Defining $\Delta \mathbf{F}^{T}=\left[\begin{array}{ll}\mathbf{0} & \Delta \mathbf{L}^{T}\end{array}\right]$ and incorporating the dynamic model for the Gauss-Markov states results in the following propagation equation

$$
\left[\begin{array}{c}
\dot{\boldsymbol{\varepsilon}} \\
\dot{\boldsymbol{a}}
\end{array}\right]=\left[\begin{array}{cc}
\hat{\mathbf{F}}(t) & \Delta \mathbf{F} \\
\mathbf{0} & \mathbf{L}
\end{array}\right]\left[\begin{array}{l}
\boldsymbol{\varepsilon} \\
\boldsymbol{a}
\end{array}\right]+\left[\begin{array}{l}
\boldsymbol{q}(t) \\
\boldsymbol{u}(t)
\end{array}\right]
$$

The estimate vector after the measurement update is $\widehat{\boldsymbol{x}}_{k \mid k}=\widehat{\boldsymbol{x}}_{k \mid k-1}+\mathbf{K}_{k}\left(\mathbf{z}_{k}-\mathbf{H}_{k} \widehat{\boldsymbol{x}}_{k \mid k-1}\right)$ such that $\mathbf{K}_{k}$ is the Kalman gain matrix. It is straightforward to show that the estimate error vector is $\boldsymbol{\varepsilon}_{k \mid k}=\left(\mathbf{I}-\mathbf{K}_{k} \mathbf{H}_{k}\right) \boldsymbol{\varepsilon}_{k \mid k-1}-\mathbf{K}_{k} \mathbf{D}_{k} \boldsymbol{n}_{v, k}$. When combined with $\boldsymbol{a}_{k}=\boldsymbol{a}_{k}$, the update equation is

$$
\left[\begin{array}{c}
\boldsymbol{\varepsilon}_{k \mid k} \\
\boldsymbol{a}_{k}
\end{array}\right]=\left[\begin{array}{cc}
\mathbf{I}-\mathbf{K}_{k} \mathbf{H}_{k} & \mathbf{0} \\
\mathbf{0} & \mathbf{I}
\end{array}\right]\left[\begin{array}{c}
\boldsymbol{\varepsilon}_{k \mid k-1} \\
\boldsymbol{a}_{k}
\end{array}\right]-\left[\begin{array}{c}
\mathbf{K}_{k} \mathbf{D}_{k} \\
\mathbf{0}
\end{array}\right] \boldsymbol{n}_{v, k}
$$

The covariance matrix $\mathbf{P}=E\left[\boldsymbol{e} \boldsymbol{e}^{T}\right]$ propagates between measurements according to [11]

$$
\begin{aligned}
& \dot{\mathbf{P}}=\left[\begin{array}{cc}
\hat{\mathbf{F}}(t) & \Delta \mathbf{F} \\
\mathbf{0} & \mathbf{L}
\end{array}\right] \mathbf{P}+\mathbf{P}\left[\begin{array}{cc}
\hat{\mathbf{F}}^{T}(t) & \mathbf{0} \\
\Delta \mathbf{F}^{T} & \mathbf{L}^{T}
\end{array}\right]+\mathbf{Q}(t), t \in\left[t_{k-1}, t_{k}\right] \\
& \mathbf{P}\left(t_{k-1}\right)=\mathbf{P}_{k-1 \mid k-1}
\end{aligned}
$$

where

$$
\mathbf{Q}(t)=E\left\{\left[\begin{array}{c}
\mathbf{B}(t) \boldsymbol{n}_{w}(t) \\
\boldsymbol{u}(t) \\
\boldsymbol{u}(t)
\end{array}\right]\left[\begin{array}{lll}
\boldsymbol{n}_{w}^{T}(t) \mathbf{B}^{T}(t) & \boldsymbol{u}^{T}(t) & \boldsymbol{u}^{T}(t)
\end{array}\right]\right\}=\left[\begin{array}{ccc}
\mathbf{B}(t) \mathbf{N}(t) \mathbf{B}^{T}(t) & \mathbf{0} & \mathbf{0} \\
\mathbf{0} & \mathbf{U} & \mathbf{U} \\
\mathbf{0} & \mathbf{U} & \mathbf{U}
\end{array}\right]
$$

During a measurement update, the covariance matrix is updated through

$$
\mathbf{P}_{k \mid k}=\left[\begin{array}{cc}
\mathbf{I}-\mathbf{K}_{k} \mathbf{H}_{k} & \mathbf{0} \\
\mathbf{0} & \mathbf{I}
\end{array}\right] \mathbf{P}_{k \mid k-1}\left[\begin{array}{cc}
\left(\mathbf{I}-\mathbf{K}_{k} \mathbf{H}_{k}\right)^{T} & \mathbf{0} \\
\mathbf{0} & \mathbf{I}
\end{array}\right]+\left[\begin{array}{c}
\mathbf{K}_{k} \mathbf{D}_{k} \mathbf{R}_{k} \mathbf{D}_{k}^{T} \mathbf{K}_{k}^{T} \\
\mathbf{0}
\end{array}\right]
$$

\section{Appendix B: Discretization of Bounding Gauss-Markov Model}

This appendix derives the discrete-time form of Eq. (21), restated below for convenience

$$
\dot{a}=-\frac{1}{\tau_{\max }} a+\sqrt{\frac{2 \sigma_{\max }^{2}}{\tau_{\min }}} w(t), \begin{aligned}
& w(t) \sim N(0,1) \\
& E[w(t) w(s)]=\delta(t-s)
\end{aligned}
$$


Denoting the discrete-time sampling interval as $\Delta t$, the corresponding discrete-time model is $a_{k+1}=\exp \left(-\Delta t / \tau_{\max }\right)+w_{k}$, where $w_{k} \sim N\left(0, \sigma_{w}^{2}\right)$. Equation (3.9.25) in [20] shows that $\sigma_{w}^{2}=$ $\exp \left(-\Delta t / \tau_{\max }\right)[\exp (\mathbf{A})]_{12}$ such that

$$
\mathbf{A}=\left[\begin{array}{cc}
1 / \tau_{\max } & 2 \sigma_{\max }^{2} / \tau_{\min } \\
0 & -1 / \tau_{\max }
\end{array}\right] \Delta t
$$

and $[\exp (\mathbf{A})]_{12}$ is the $(1,2)$ element of the matrix exponential of $\mathbf{A}$. It can be shown analytically that

$$
[\exp (\mathbf{A})]_{12}=\frac{\sigma_{\max }^{2} \tau_{\max }}{\tau_{\min }}\left[\exp \left(\Delta t / \tau_{\max }\right)-\exp \left(-\Delta t / \tau_{\max }\right)\right]
$$

which leads to

$$
\sigma_{w}^{2}=\frac{\sigma_{\max }^{2} \tau_{\max }}{\tau_{\min }}\left[1-\exp \left(-2 \Delta t / \tau_{\max }\right)\right]
$$

The discrete-time form of Eq. (B1) is thus given by

$$
a_{k+1}=\exp \left(-\frac{\Delta t}{\tau_{\max }}\right) a_{k}+\sqrt{\sigma_{\max }^{2}\left(\frac{\tau_{\max }}{\tau_{\min }}\right)\left[1-\exp \left(-\frac{2 \Delta t}{\tau_{\max }}\right)\right]} w_{k} \begin{aligned}
& w_{k} \sim N(0,1) \\
& E\left[w_{k} w_{l}\right]=\delta_{k l}
\end{aligned}
$$

\section{Appendix C: Minimum Allowable Initial Variance}

This appendix derives the minimum allowable initial variance for the process in Eq. (25). The derivation is based on the requirement that $\mathbf{E}(0) \geq 0$ and $\boldsymbol{\Sigma}_{x a}(0)=\mathbf{0}$. If no prior information exists to initialize $\boldsymbol{a}$ (the case considered here), the initial estimate for the augmented states is $\widehat{\boldsymbol{a}}_{0}=$ 0. This leads to the following expected values

$$
\begin{aligned}
E\left[\boldsymbol{\varepsilon}_{\xi, 0} \boldsymbol{\varepsilon}_{a, 0}^{T}\right] & =\mathbf{0} \\
E\left[\boldsymbol{\varepsilon}_{\xi, 0} \boldsymbol{\varepsilon}_{a, 0}^{T}\right] & =E\left[\boldsymbol{\varepsilon}_{\xi, 0}\left(\boldsymbol{a}_{0}-\widehat{\boldsymbol{a}}_{0}\right)^{T}\right]=E\left[\boldsymbol{\varepsilon}_{\xi, 0} \boldsymbol{a}_{0}^{T}\right]=\mathbf{0} \\
E\left[\boldsymbol{\varepsilon}_{a, 0} \boldsymbol{\varepsilon}_{a, 0}^{T}\right] & =E\left[\left(\boldsymbol{a}_{0}-\widehat{\boldsymbol{a}}_{0}\right)\left(\boldsymbol{a}_{0}-\widehat{\boldsymbol{a}}_{0}\right)^{T}\right]=E\left[\boldsymbol{a}_{0} \boldsymbol{a}_{0}^{T}\right] \\
E\left[\boldsymbol{\varepsilon}_{a, 0} \boldsymbol{a}_{0}^{T}\right] & =E\left[\left(\boldsymbol{a}_{0}-\widehat{\boldsymbol{a}}_{0}\right) \boldsymbol{a}_{0}^{T}\right]=E\left[\boldsymbol{a}_{0} \boldsymbol{a}_{0}^{T}\right]
\end{aligned}
$$

where we have used the fact that $E\left[\boldsymbol{\varepsilon}_{\xi, 0} \widehat{\boldsymbol{a}}_{0}^{T}\right]=E\left[\boldsymbol{a}_{0} \widehat{\boldsymbol{a}}_{0}^{T}\right]=E\left[\widehat{\boldsymbol{a}}_{0} \widehat{\boldsymbol{a}}_{0}^{T}\right]=\mathbf{0}$ because $\widehat{\boldsymbol{a}}_{0}=\mathbf{0}$. Using the relations in Eq. (C1), the true initial covariance matrix is

$$
\mathbf{P}(0)=E\left\{\left[\begin{array}{c}
\boldsymbol{\varepsilon}_{\xi, 0} \\
\boldsymbol{\varepsilon}_{a, 0} \\
\boldsymbol{a}_{0}
\end{array}\right]\left[\begin{array}{lll}
\boldsymbol{\varepsilon}_{\xi, 0}^{T} & \boldsymbol{\varepsilon}_{a, 0}^{T} & \boldsymbol{a}_{0}
\end{array}\right]\right\}=\left[\begin{array}{ccc}
\mathbf{P}_{\xi, 0} & \mathbf{0} & \mathbf{0} \\
\mathbf{0} & \mathbf{P}_{a, 0} & \mathbf{P}_{a, 0} \\
\mathbf{0} & \mathbf{P}_{a, 0}^{T} & \mathbf{P}_{a, 0}
\end{array}\right]
$$


The requirement $\boldsymbol{\Sigma}_{x a}(0)=\mathbf{0}$ implies that

$$
\boldsymbol{\Sigma}(0)=\left[\begin{array}{ccc}
\boldsymbol{\Sigma}_{\xi, 0} & \mathbf{0} & \mathbf{0} \\
\mathbf{0} & \boldsymbol{\Sigma}_{a, 0} & \mathbf{0} \\
\mathbf{0} & \mathbf{0} & \overline{\boldsymbol{\Sigma}}_{a, 0}
\end{array}\right]
$$

where $\overline{\boldsymbol{\Sigma}}_{a, 0}$ is a diagonal matrix consisting of the $\bar{\sigma}_{l}^{2}$ in Eq. (21). The goal is to determine $\boldsymbol{\Sigma}_{a, 0}$ so that $\boldsymbol{\Sigma}(0) \geq \mathbf{P}(0)$. Subtracting $\mathbf{P}(0)$ from $\boldsymbol{\Sigma}(0)$ results in

$$
\boldsymbol{\Sigma}(0)-\mathbf{P}(0)=\left[\begin{array}{ccc}
\boldsymbol{\Sigma}_{\xi, 0}-\mathbf{P}_{\xi, 0} & \mathbf{0} & \mathbf{0} \\
\mathbf{0} & \boldsymbol{\Sigma}_{a, 0}-\mathbf{P}_{a, 0} & -\mathbf{P}_{a, 0} \\
\mathbf{0} & -\mathbf{P}_{a, 0}^{T} & \overline{\boldsymbol{\Sigma}}_{a, 0}-\mathbf{P}_{a, 0}
\end{array}\right]
$$

Using Schur complements, [21] shows that $\boldsymbol{\Sigma}(0) \geq \mathbf{P}(0)$ if and only if

$$
\left[\begin{array}{cc}
\boldsymbol{\Sigma}_{\xi, 0}-\mathbf{P}_{\xi, 0} & \mathbf{0} \\
\mathbf{0} & \boldsymbol{\Sigma}_{a, 0}-\mathbf{P}_{a, 0}
\end{array}\right]-\left[\begin{array}{c}
\mathbf{0} \\
\mathbf{P}_{a, 0}
\end{array}\right]\left(\overline{\boldsymbol{\Sigma}}_{a, 0}-\mathbf{P}_{a, 0}\right)^{-1}\left[\begin{array}{ll}
\mathbf{0} & \mathbf{P}_{a, 0}^{T}
\end{array}\right] \geq 0
$$

or

$$
\left[\begin{array}{cc}
\boldsymbol{\Sigma}_{\xi, 0}-\mathbf{P}_{\xi, 0} & \mathbf{0} \\
\mathbf{0} & \left(\boldsymbol{\Sigma}_{a, 0}-\mathbf{P}_{a, 0}\right)-\mathbf{P}_{a, 0}\left(\overline{\boldsymbol{\Sigma}}_{a, 0}-\mathbf{P}_{a, 0}\right)^{-1} \mathbf{P}_{a, 0}^{T}
\end{array}\right] \geq 0
$$

It is assumed that $\boldsymbol{\Sigma}_{\xi, 0}$ can be determined so that $\left(\boldsymbol{\Sigma}_{\xi, 0}-\mathbf{P}_{\xi, 0}\right) \geq 0$. Then it suffices to show that

$$
\left(\boldsymbol{\Sigma}_{a, 0}-\mathbf{P}_{a, 0}\right)-\mathbf{P}_{a, 0}\left(\overline{\boldsymbol{\Sigma}}_{a, 0}-\mathbf{P}_{a, 0}\right)^{-1} \mathbf{P}_{a, 0}^{T} \geq 0
$$

All matrices in Eq. (C7) are diagonal. Thus, we can consider the scalar condition

$$
\left(\sigma_{0}^{2}-\sigma^{2}\right)-\frac{\sigma^{4}}{\bar{\sigma}^{2}-\sigma^{2}} \geq 0
$$

Substituting the result from Eq. (21) and simplifying yields

$$
\sigma_{0}^{2} \geq \frac{2 \sigma^{2}}{1+\left(\tau / \tau_{\max }\right)}
$$

With $\sigma^{2} \in\left[\sigma_{\min }^{2}, \sigma_{\max }^{2}\right]$ and $\tau \in\left[\tau_{\min }, \tau_{\max }\right]$, the initial variance $\sigma_{0}^{2}$ must satisfy the inequality

$$
\sigma_{0}^{2} \geq \frac{2 \sigma_{\max }^{2}}{1+\left(\tau_{\min } / \tau_{\max }\right)}
$$

